\title{
Cross-modal signaling in giant pandas
}

\author{
XU Meng ${ }^{1}$, WANG ZhiPeng ${ }^{1}$, LIU DingZhen ${ }^{1 *}$, WEI RongPing ${ }^{2}$, ZHANG GuiQuan $^{2}$, \\ ZHANG HeMin ${ }^{2}$, ZHOU XiaoPing ${ }^{2} \&$ LI DeSheng ${ }^{2}$ \\ ${ }^{1}$ Key Laboratory of Biodiversity Science and Ecological Engineering of Ministry of Education, College of Life Sciences, Beijing Normal \\ University, Beijing 100875, China; \\ ${ }^{2}$ Key Laboratory for Reproduction and Conservation Genetics of Endangered Wildlife of Sichuan, China Conservation and Research Center \\ for the Giant Panda, Wolong 623006, China
}

Received August 3, 2011; accepted September 23, 2011

\begin{abstract}
For solitary species that aggregate to breed, signals, such as sound or odor, transmitted across the landscape can play an important role in mate choice and coordinating breeding activity. Recent work on vocalizations of the giant panda (Ailuropoda melanoleuca), bleats and chirps that are emitted during periods of reproductive activity, has revealed that auditory communication in this species is more complicated and developed than once believed. However, playback experiments using these calls have recorded only a few behaviors over short observation times and the influence of these calls on the signaling behavior of receivers remains unknown. Here, we present results from a pilot study in which we played the bleats of male and female giant pandas in estrus to captive animals and measured vocal and chemical signaling response behavior. We found an increase in scent marking behavior, but not vocalizations, in males and females following the playback of calls made by members of the opposite sex in estrus. To our knowledge, this is the first study to detect a chemical communication response, which was marking, to an auditory signal in giant pandas. Our findings reveal a previously unknown relationship between acoustic and chemical signals in this high profile species and suggest that these two forms of signaling may be interdependent in the social lives of giant pandas.
\end{abstract}

\section{Ailuropoda melanoleuca, auditory communication, chemical communication, multiple modality, giant panda}

Citation: $\quad$ Xu M, Wang Z P, Liu D Z, et al. Cross-modal signaling in giant pandas. Chin Sci Bull, 2012, 57: 344-348, doi: 10.1007/s11434-011-4843-y

Vocalizations are an important method used by animals to communicate [1]. Research has revealed a range of languages, cultures, functions and social contexts in which vocalizations are fundamental in a wide range of species [2-6]. Research on auditory signals can take an acoustic, behavioral or functional approach, or a combination of these $[7,8]$. Auditory communication in mammals has been shown to have a range of functions. For example, individuals detect, find and choose mates based on the vocalizations of others in free ranging yellow baboons, Papio cynocephalus cynocephalus $[9,10]$. Males use calls to deter rivals when defending territory and competing for access to females and other resources in ungulates, such as red deer Cervus elaphus and fallow bucks Dama dama [11,12]. Sound can also be used as an alarm in social animals, such

*Corresponding author (email: dzliu@ bnu.edu.cn) as ground-dwelling Sciurids and primates [13,14]. Vocal signals are also used by gregarious mammals to maintain social bonds, such as for parent-offspring recognition [15-18].

For solitary species that only aggregate to breed, signals, such as sound (audible and ultrasonic song), body display and odor, transmitted across the landscape can play an important role in mate choice and coordinating breeding activity $[12,19,20]$. Male and female animals often switch from one communication modality to another in response to courtship calls, body display or scent marking during the mating season. For example, female concave-eared torrent frogs (Odorrana tormota) make ultrasonic calls instead of audible calls to communicate with courtship males immediately before ovulation [21]. Female swamp sparrows (Melospiza georgiana) display significantly more solicitation responses to high-performance versus low-performance 
songs of the same song type produced by different males [22]. The issue of cross-modalities communication and mate choice is an evolving area of research, and even in highprofile species our understanding of these systems is poor. Recent work on giant pandas (Ailuropoda melanoleuca) [23-26] has revealed that auditory communication is more complicated than previously believed. Giant pandas are solitary animals that produce 11 different vocalizations across a range of contexts, some of which change in rate and structure during periods of reproductive activity [27,28]. Male and female giant pandas frequently produce vocalizations (bleats and chirps) and deposit scent markings during the mating season [29]. Bleats and chirps are nonaggressive and affinitive calls predominately used in sexual attraction and the synchronization of estrus and mating in giant pandas [30-33]. Acoustic analysis has shown that bleats are individualized and may signal caller sex, age and body size [23]. In addition, female giant panda chirps can signal their precise estrus state [24]. Experiments involving the playback of male calls to females has shown that female giant pandas can discriminate between the vocalizations of males and show preference to potential mates [23]. Male giant pandas can also perceive differences in female chirps and possibly also the time of female fertile phases [24]. Giant panda calls are often produced alongside increases in marking behavior $[34,35]$ and while marking and vocalization behavior have been studied separately, the relationship between these signaling modes remains unknown. Here we present results from a pilot study using captive giant pandas that focused on the auditory and olfactory responses to the bleats of male and female giant pandas during estrus. This research will add to our growing appreciation of communication in giant pandas and other large mammals, and may have applications for the captive management of this iconic species.

\section{Materials and methods}

\subsection{Animals}

We conducted this study at the China Conservation and Research Center for the Giant Panda at Wolong Nature Reserve, Sichuan, China. Our study comprised 10 adult females and 8 adult males aged 5-16 years (Table 1) and took place from March to June 2005. Focal males and females were examined during estrus. At this facility, animals are individually housed in typical indoor/outdoor enclosures [36] that are cleaned daily from 07:30-09:00 hours. Animals were fed twice daily (morning and afternoon) fresh bamboo and a mix of corn, bamboo powder, soybean and egg.

\subsection{Recordings and playback stimuli}

Male bleats were recorded from Wugang during estrus and female bleats were recorded from Maomao during estrus
Table 1 Subjects used in the pilot study ${ }^{\text {a) }}$

\begin{tabular}{|c|c|c|c|}
\hline Name & Studbook number & $\begin{array}{c}\text { Age in years during } \\
\text { experiment }\end{array}$ & Gender \\
\hline Zhuangzhuang & 357 & 16 & Male \\
\hline Yingying & 369 & 14 & Male \\
\hline Ximeng & 399 & 12 & Male \\
\hline Lingling & 424 & 10 & Male \\
\hline Linlin & 455 & 8 & Male \\
\hline Yuanyuan & 488 & 6 & Male \\
\hline Wugang & 502 & 6 & Male \\
\hline Lulu & 503 & 6 & Male \\
\hline Leilei & 374 & 16 & Female \\
\hline$\# 20$ & 414 & 15 & Female \\
\hline Haizi & 544 & 11 & Female \\
\hline Feifei & 432 & 10 & Female \\
\hline Guoguo & 439 & 9 & Female \\
\hline Youyou & 474 & 7 & Female \\
\hline Gongzhu & 477 & 7 & Female \\
\hline Maomao & 504 & 6 & Female \\
\hline Yeye & 495 & 6 & Female \\
\hline Longxin & 516 & 5 & Female \\
\hline
\end{tabular}

a) As of 2005.

from March to June 2005. Wugang and Maomao are not genetically related to our subjects, did not engage in abnormal behavior and showed normal signs of fertility. Calls were captured using a Sony ICD-SX40 MP3 player (Sony, Tokyo, Japan) and Samson S11 microphone (Samson Technologies, Hauppauge, United States). All sounds were recorded at maximum microphone power. We recorded 10-30 calls from each animal during scent marking behavior and saved them in MP3 format. We chose calls that contained the least background noise for use as playback stimuli. Stimuli consisted of 10 bleats (frequency: $60 \mathrm{~Hz}-$ $13.5 \mathrm{kHz}$ ) each separated by $10 \mathrm{~s}$ and were played using a Sony ICD-SX40 MP3 player (Sony) with a $70 \mathrm{~mW}$ speaker at maximum volume. The MP3 player was hidden from the focal animal behind a fixture within the enclosure.

\subsection{Behavioral responses to playbacks}

Playbacks were conducted between 8:00-10:30 and 14:00-16:30 hours. Before each observation, the door connecting the indoor and outdoor sections of the enclosure was opened and the animal was enticed inside by animal keepers. To reduce the possible effects of neighboring individual's chemical, auditory and visual signal stimuli, the subject was kept inside the indoor section of the enclosure by closing the door connecting the indoor and outdoor sections while the tests were being run. After entering the indoor area, animals were given $3 \mathrm{~min}$ to adapt to the area before a stimulus was played or the control treatment commenced. We chose 3 min because in our experience this is the average time that a giant panda inspects a chamber after 
entering and before engaging in stereotypical behavior. For the playback, we played bleats from Wugang to 10 females in estrus and bleats from Maomao to 8 males in estrus. Focal animals were observed for $10 \mathrm{~min}$. The same playback stimulus was played to each focal animal 3 times, separated by $2 \mathrm{~d}$ (range: $2-3 \mathrm{~d}$ ). A control treatment consisted of observing the animal for $10 \mathrm{~min}$ with the speaker power on and at maximum volume, but with no input. Control treatments were run only once for each focal animal. For each subject, the test lasted $40 \mathrm{~min}$, which included one 10-min control test and three 10-min playback tests. Thus, for the 18 animals (10 female, 8 male), 720 min of observation and video recordings were collected. The focal animal sampling method and continuous recording method were used for the behavioral observations.

Our subjects were housed within a breeding and research center and have experienced long-term cohabitation within its confines. Given the physical space between enclosures and degree of separation between animals, we doubt that our focal animals have developed a preference for our particular donor male or female based on vocalizations. We chose donors inhabiting enclosures as far as possible from focal animals; we cannot however, rule out the possibility that our focal animals had developed an acoustic memory of the donor animals (Wugang and Maomao) used in the playbacks.

\subsection{Behavioral and statistical analyses}

We noted all marking and vocalization behavior during the observations. We defined marking behavior as rubbing the anogenital gland against a surface. For vocalization behavior, we noted the number of bleats emitted. Behaviors were recorded in real-time by a single trained observer positioned 2-3 m from the enclosure with an unobstructed view of the focal animal. Observers were aware of the identity of the donor and familiar with the behavior of giant pandas in captivity. For our analyses, we tabulated the frequency of marking and bleating across all three playback trials and three control treatments and then averaged these values to obtain one value for the playback and one for the control. We inspected the distribution of the data for normality. To investigate evidence for an effect of the playback, we used paired-sample $t$ tests in SPSS for Windows 13.0 (SPSS Inc, Chicago, United States). The significance level was 0.05 and all tests were two-tailed.

\section{Results}

Male giant pandas increased marking behavior following the playback of Maomao's bleat (paired $t$ test, $d f=7, P=$ 0.001 ) and females increased marking behavior following exposure to Wugang's bleats (paired $t$ test, $d f=9, P=0.01$ ). No change in the number of bleats emitted by focal males and females following the playbacks was observed (female: $d f=9, P=0.293$; male: $d f=7, P=0.300$; Figure 1 ).

\section{Discussion}

Chemical and auditory communication are major modes of signaling in the lives of giant pandas [33]. Giant panda male and female urine and male anogenital gland secretions contain information that allow individuals to discriminate age, gender and social status [34,37-41]. We have a basic understanding of chemical signaling in giant pandas [37,38], and recent work on their vocal communications has raised the profile of this aspect of their sociality [23,25]. It is now clear that a great deal of information is coded in the bleats and chirps of giant pandas and ours is the first study to detect a chemical communication response, which was marking, to these auditory signals. This association between chemical and vocal signaling is further supported by work on captive and wild giant pandas $[30,33,42]$. For example, adults produce more bleats, chirps and markings when are translocated to an enclosure belonging to the opposite sex $[34,35]$ and in captivity male and female giant panda switch from scent marking to chirping and bleating as the breeding season progresses [43]. This mix and switch of auditory and chemical communication is common in captive giant pandas, but data from the wild is still lacking $[33,44]$. Our findings have shown a clear relationship between cross-modal signals that will hopefully stimulate further research in both the giant panda and other mammals.

One of the goals of the giant panda captive breeding program is to produce healthy individuals with species-typical behavior, such as mating and foraging. However, more than half of annual breeding output is realized via artificial insemination or a combination of artificial insemination and natural mating (Liu D, unpublished data). Further, some
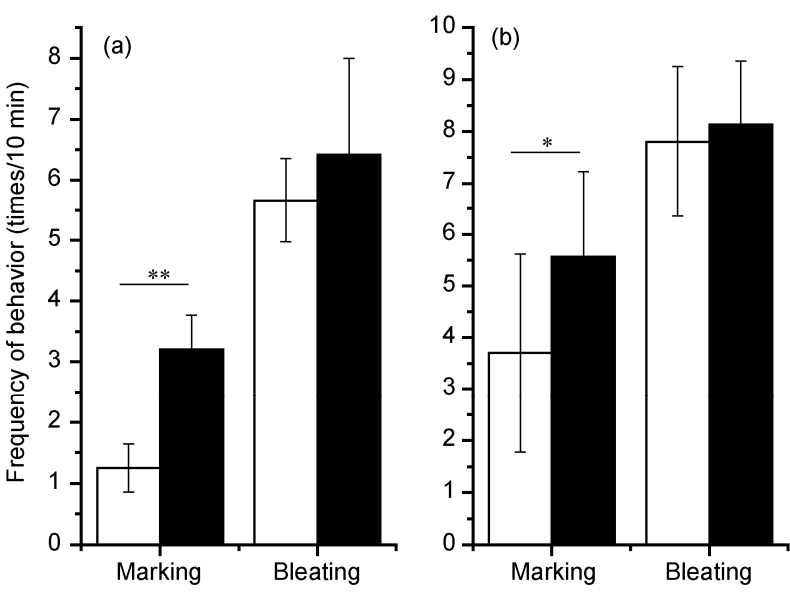

Figure 1 Marking and bleating frequency (times/10 min) of (a) male giant pandas to the bleats of estrus female (black bars) and (b) female giant pandas to the bleats of estrus males (black bars) and a control treatment (white bars). Data are mean \pm SE. ${ }^{*} P<0.05$, ** $P<0.01$. 
adult males are unable to mate naturally with females because of asynchrony or female sexual inactivity [29]. These issues may be because of a focus on the chemical environment $[34,39,45]$ and a lack of understanding of the role of vocal communication in giant panda mate selection and reproduction. Recent acoustic analyses in combination with our findings suggest auditory communication may play the same role as chemical communication in the social lives of giant pandas [23-25]. Further work is needed before we can apply our understanding of vocal signaling to giant panda captive breeding programs. Our limited number of sound donors means that we are unable to generalize further about the role of vocal signaling in this species, but the exciting finding of cross-modal signaling in this species should guide future research into wild and captive giant pandas.

We are grateful to the China Conservation and Research Center for the Giant Panda for their help in conducting this experiment and Dr Zuoxin Wang for advice. This work was supported by the National Natural Science Foundation of China (30470233 and 31170297) and the International Cooperative Project for the Giant Panda, State Forestry Administration (WH0306, WH0309).

1 Krebs J, Davies N, Parr J. An Introduction to Behavioural Ecology. 3rd edn. London: Wiley-Blackwell, 1993. 349-374

2 Hollen L I, Radford A N. The development of alarm call behaviour in mammals and birds. Anim Behav, 2009, 78: 791-800

3 Marler P, Slabbekoorn H. Nature's Music: The Science of Birdsong. New York: Academic Press, 2004

4 Naguib M, Janik V, Clayton N, et al. Vocal Communication in Birds and Mammals. New York: Academic Press, 2009

5 Stumpner A, Von Helversen D. Evolution and function of auditory systems in insects. Naturwissenschaften, 2001, 88: 159-170

6 Vergne A, Pritz M, Mathevon N. Acoustic communication in crocodilians: From behaviour to brain. Biol Rev, 2009, 84: 391-411

7 Johnson M, de Soto N, Madsen P. Studying the behaviour and sensory ecology of marine mammals using acoustic recording tags: A review. Mar Ecol Prog Ser, 2009, 395: 55-73

8 Taylor A, Reby D. The contribution of source-filter theory to mammal vocal communication research. J Zool, 2009, 280: 221-236

9 Semple $\mathrm{S}$. Individuality and male discrimination of female copulation calls in the yellow baboon. Anim Behav, 2001, 61: 1023-1028

10 Semple S, McComb K, Alberts S, et al. Information content of female copulation calls in yellow baboons. Am J Primatol, 2002, 56: 43-56

11 McComb K E. Female choice for high roaring rates in red deer, $\mathrm{Cer}$ vus elaphus. Anim Behav, 1991, 41: 79-88

12 McElligott A G, O’Neill K P, Hayden T J. Cumulative long-term investment in vocalization and mating success of fallow bucks, Dama dama. Anim Behav, 1999, 57: 1159-1167

13 Macedonia J M, Evans C S. Variation among mammalian alarm call systems and the problem of meaning in animal signals. Ethology, 1993, 93: 177-197

14 Sherman P W. Nepotism and the evolution of alarm calls. Science, 1977, 197: 1246-1253

15 Caudron A K, Kondakov A A, Siryanov S V. Acoustic structure and individual variation of grey seal (Halichoerus grypus) pup calls. J Mar Biol Assoc UK, 1998, 78: 651-658

16 Charrier I, Mathevon N, Jouventin P. How does a fur seal mother recognize the voice of her pup? An experimental study of Arctocephalus tropicalis. J Exp Biol, 2002, 205: 603-612

17 Collins K T, Terhune J M, Rogers T L, et al. Vocal individuality of in-air weddell seal (Leptonychotes weddellii) pup "primary" calls. Mar Mammal Sci, 2006, 22: 933-951

18 Phillips A V, Stirling I. Vocal individuality in mother and pup South American fur seals, Arctocephalus australis. Mar Mammal Sci, 2000, 16: 592-616

19 Bradbury J W, Vehrencamp S L. Principles of Animal Communication. Sunderland, MA: Sinauer Associates, 1998

20 Eisenberg J F, Kleiman D G. Olfactory communication in mammals. Annu Rev Ecol Syst, 1972, 3: 1-32

21 Shen J X, Feng A S, Xu Z M, et al. Ultrasonic frogs show hyperacute phonotaxis to female courtship calls. Nature, 2008, 453: 914-916

22 Ballentine B, Hyman J, Nowicki S. Vocal performance influences female response to male bird song: An experimental test. Behav Ecol, 2004, 15: 163-168

23 Charlton B D, Huang Y, Swaisgood R R. Vocal discrimination of potential mates by female giant pandas (Ailuropoda melanoleuca). Biol Lett, 2009, 5: 597-599

24 Charlton B D, Keating J L, Li R G, et al. Female giant panda (Ailuropoda melanoleuca) chirps advertise the caller's fertile phase. P Roy Soc B-Biol Sci, 2010, 277: 1101-1106

25 Charlton B D, Zhang Z H, Snyder R J. Vocal cues to identity and relatedness in giant pandas (Ailuropoda melanoleuca). J Acoust Soc Am, 2009, 126: 2721-2732

26 Charlton B D, Zhang Z H, Snyder R J. The information content of giant panda, Ailuropoda melanoleuca, bleats: Acoustic cues to sex, age and size. Anim Behav, 2009, 78: 893-898

27 Peters G. A comparative survey of vocalization in the giant panda, Ailuropoda melanoleuca (David 1869). In: Klös H G, Frädrich H, eds. Proceedings of the International Symposium on the Giant Panda. Berlin, Germany, 1985. 197-208

28 Zhu J, Meng Z B. On the vocal behavior during the estrous period of the giant panda, Ailuropoda melanoleuca (in Chinese). Acta Zool Sin, 1987, 33: 285-292

29 Liu D, Fang J, Sun R, et al. Behavioral comparison in individuals of different sexual ability in giant panda (Ailuropoda melanoleuca) (in Chinese). Acta Zool Sin, 1998, 44: 27-34

30 Kleiman D G. Ethology and reproduction of captive giant pandas (Ailuropoda melanoleuca). Z Tierpsychol, 1983, 62: 1-46

31 Kleiman D G, Peters G. Auditory communication in the giant panda: Motivation and function. In: Asakura S, Nakagawa S, eds. Proceedings of the Second International Symposium on Giant Panda. Tokyo: Tokyo Zoological Park Society, 1990. 107-122

32 Peters G. A note on the vocal behavior of the giant panda (Ailuropoda melanoleuca). Int J Mamm Biol, 1982, 47: 236-246

33 Schaller G B, Hu J, Pan W, et al. The Giant Pandas of Wolong. Chicago: University of Chicago Press, 1985

34 Liu D, Zhang G, Wei R, et al. Behavioral responsiveness of captive giant pandas (Ailuropoda melanoleuca) to substrate odors from conspecifcs of the opposite sex. In: Mason R T, LeMaster M P, Müller-Schwarze D, eds. Chemical Signals in Vertebrates 10. New York: Springer, 2005. 101-109

35 Swaisgood R, Lindburg D, Zhou X, et al. The effects of sex, reproductive condition and context on discrimination of conspecific odours by giant pandas. Anim Behav, 2000, 60: 227-237

36 Liu D, Wang Z, Tian H, et al. Behavior of giant pandas (Ailuropoda melanoleuca) in captive conditions: Gender differences and enclosure effects. Zoo Biol, 2003, 22: 77-82

37 Liu D, Yuan H, Tian H, et al. Do anogenital gland secretions of giant panda code for their sexual ability? Chin Sci Bull, 2006, 51: 1986-1995

38 Liu D, Wei R P, Zhang G Q, et al. Male panda (Ailuropoda melanoleuca) urine contains kinship information. Chin Sci Bull, 2008, 53: 2793-2800

39 Swaisgood R R, Lindburg D G, Zhang H. Discrimination of oestrous status in giant pandas (Ailuropoda melanoleuca) via chemical cues in urine. J Zool, 2002, 257: 381-386

40 Tian H, Wei R P, Zhang G Q, et al. Age differences in behavioral responses of male giant pandas to chemosensory stimulation. Zool Res, 2007, 28: 134-140 
41 White A M, Swaisgood R R, Zhang H. Urinary chemosignals in giant pandas (Ailuropoda melanoleuca): Seasonal and developmental effects on signal discrimination. J Zool, 2004, 264: 231-238

42 Liu D, Zhang G, Wei R, et al. The effects of sex and age on the behavior of captive giant pandas (Ailuropoda melanoleuca) (in Chinese). Acta Zool Sin, 2002, 48: 585-590

43 Zhang $\mathrm{H} \mathrm{M}$, Wang P Y, Zhang G Q, et al. Advances in conservation and research technology for captive and wild pandas of Wolong. Si- chuan J Zool, 2000, 19: 35-38

44 Kleiman D G. Social and reproductive behaviors of the giant panda (Ailuropoda melanoleuca). In: Klös H G, Frädrich H, eds. Proceedings of the International Symposium on the Giant Panda. Berlin, Germany, 1985. 45-58

45 Swaisgood R R, Lindburg D G, Zhou X P. Giant pandas discriminate individual differences in conspecific scent. Anim Behav, 1999, 57: 1045-1053

Open Access This article is distributed under the terms of the Creative Commons Attribution License which permits any use, distribution, and reproduction in any medium, provided the original author(s) and source are credited. 